\title{
A Chiral Interlocking Auxiliary Strategy for the Synthesis of Mechanically Planar Chiral Rotaxanes
}

Alberto de Juan," David Lozano," Andrew W. Heard, ${ }^{+}$Michael A. Jinks, ${ }^{+}$Jorge Meijide Suarez ${ }^{+}$and Stephen M. Goldup*

\#These authors contributed equally

+These authors contributed equally

*s.goldup@soton.ac.uk

Chemistry, University of Southampton, Highfield, Southampton, SO17 1BJ (UK)

\begin{abstract}
We have serendipitously discovered a combination of reaction partners that function as a "chiral interlocking auxiliary" to both orientate a macrocycle and, effectively, load it onto a new axle. We demonstrate the potential of this finding through the synthesis of a number of targets in high enantiopurity, without separation of stereoisomers, including examples whose axles lack any functional groups that would allow their direct synthesis by other means, so called "impossible" rotaxanes. Intriguingly, by varying the order of bond forming steps, we can effectively choose which end of an axle the macrocycle is loaded onto, allowing the synthesis of both hands of a single target using the same reactions and building blocks.
\end{abstract}

\section{Introduction}

The ability of the covalent subcomponents in mechanically interlocked molecules (MIMs) ${ }^{1}$ to undergo large amplitude relative motion ${ }^{2,3}$ is perhaps the most well-appreciated consequence of the mechanical bond. However, threading covalent fragments through one another to generate MIMs can also lead to molecular stereochemistry that relies solely on the mechanical bond, ${ }^{4}$ allowing MIMs to display molecular chirality even when their covalent subcomponents themselves are achiral. The synthesis of such mechanically chiral molecules in enantiopure form ${ }^{5}$ has largely relied on the chiral stationary phase HPLC (CSP-HPLC) separation of a racemic mixture of products, ${ }^{6,7,8}$ which has limited the investigation of their properties.

Indeed, to date, only two direct enantioselective syntheses of mechanically planar chiral (MPC) rotaxanes, molecules in which the stereochemistry relies on the combination of an axle that lacks mirror planes perpendicular to its principle axis and a macrocycle whose only mirror planes lie parallel to the ring, have been reported. ${ }^{9,10}$ Takata and co-workers reported the formation of MPC enantiomers in $4.4 \%$ ee using the dynamic kinetic resolution of a pseudo-rotaxane precursor. ${ }^{9 a}$ More recently, Leigh and coworkers reported a direct enantioselective synthesis of MPC rotaxanes under substrate control in an impressive $50 \%$ ee using their organocatalytic active template ${ }^{11}$ reaction ${ }^{12}$ in conjunction with a chiral alcohol leaving group. ${ }^{9 b}$ Such direct enantioselective approaches are extremely attractive but, unless $\sim 90 \%$ ee or higher can be achieved, the enantiomeric products still require CSP-HPLC separation for use in applications such as catalysis, ${ }^{13}$ sensing ${ }^{8,14}$ and materials science. ${ }^{15}$

An alternative approach to enantiopure mechanically chiral MIMs that can circumvent the need for CSPHPLC separation is to use a chiral auxiliary; ${ }^{16}$ including an enantiopure fixed covalent stereogenic unit in the MIM structure gives rise to a mixture of diastereomers that differ in the configuration of the mechanical stereogenic unit as an unequal mixture. ${ }^{17,18}$ Where required, these diastereomers can be separated using standard synthetic techniques prior to removal of the covalent stereogenic unit to provide enantioenriched mechanically chiral products in which the mechanical bond provides the sole stereogenic unit. We have demonstrated this concept in the stereoselective synthesis of enantiopure MPC rotaxanes ${ }^{17}$ and related topologically chiral catenanes. ${ }^{18}$ However, although our auxiliary approach has allowed us to demonstrate the use of MPC rotaxanes in enantioselective Au-mediated catalysis, ${ }^{13}$ the diastereoselectivity of the key mechanical bond forming step can vary significantly with the same auxiliary depending on the coupling partners used. ${ }^{17}$ Furthermore, the separation of the mechanical epimers produced is far from simple or assured. 
Here we report the serendipitous discovery of a structural motif that functions both as a chiral auxiliary, to select the orientation of a macrocycle on an axle, and an interlocking auxiliary, to effectively allow an oriented macrocycle to be threaded selectively onto an axle (Figure 1). We show that this "chiral interlocking auxiliary" approach allows the synthesis of MPC rotaxanes in excellent enantiopurity (92 $99 \%$ ee), including so-called "impossible" rotaxanes and functionalized examples through late-stage diversification. Furthermore, by effectively selecting which end of an axle the macrocycle is threaded onto, we demonstrate the synthesis of both hands of a target using the same building blocks, highlighting an intrinsic link between mechanical motion and mechanical stereochemistry.

Figure 1. The "chiral interlocking auxiliary" concept in the synthesis MPC rotaxanes

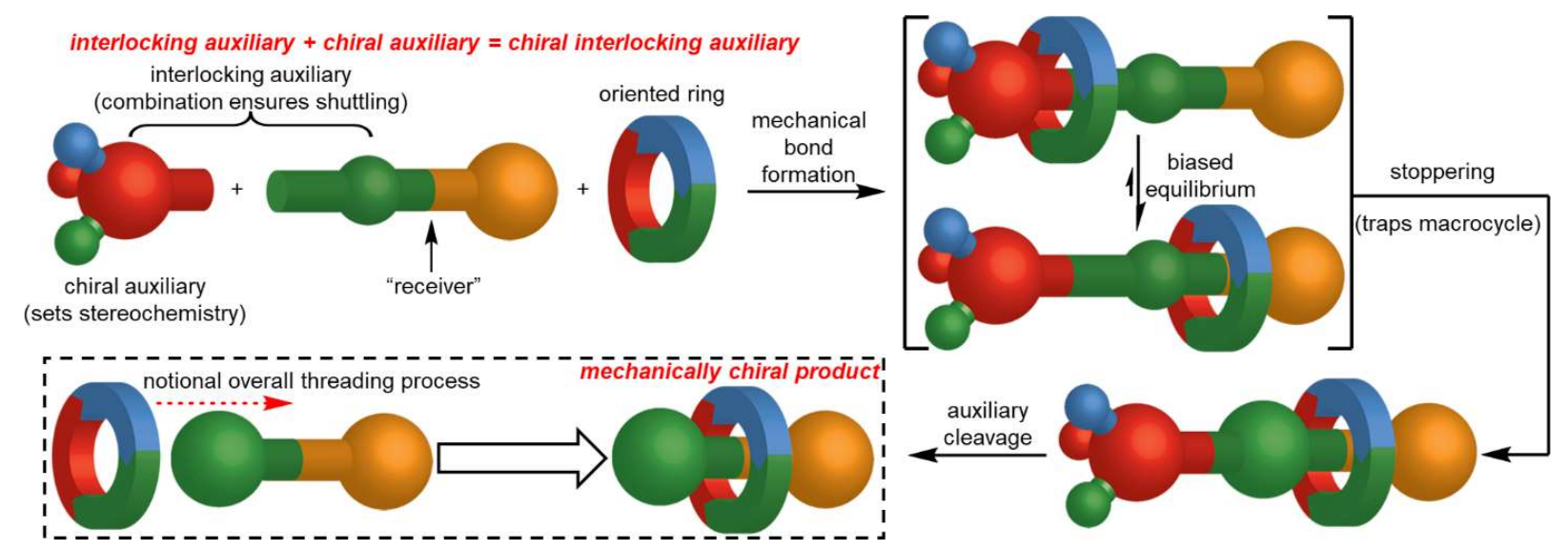

\section{Results and discussion}

\section{Discovery of a potential chiral interlocking auxiliary motif}

We previously demonstrated that amino acid-derived azide 1 can be used to generate MPC rotaxanes in up to $96 \%$ ee using an active template copper-mediated alkyne-azide cycloaddition (AT-CuAAC) ${ }^{19}$ strategy. ${ }^{17}$ However, the stereoselectivity achieved with 1 varies considerably depending on the alkyne coupling partner used. ${ }^{17}$ During efforts to understand this problem, we compared the reaction of (S)-1 in the presence of macrocycle $\mathbf{2}$ with alkynes $\mathbf{3 a}$ and $\mathbf{3 b}$, which differ in the steric hindrance around the acetylene moiety (Figure 2a). Alkyne 3a gave rise to rotaxane $\mathbf{4 a}$ in good yield but poor stereoselectivity $(76 \% d e)$, which could be assessed readily by ${ }^{1} \mathrm{H}$ NMR analysis of the crude reaction product; two resonances were observed for triazole proton $\left(\mathrm{H}_{t z z}\right)$ at 8.93 and $8.75 \mathrm{ppm}$ which were attributable to the major and minor mechanical epimers respectively (Figure 2ai). Both resonances appear at higher ppm than the corresponding signal of the non-interlocked axle ( $\Delta \delta=1.2 \mathrm{ppm}$ for the major isomer), presumably due to a $\mathrm{C}-\mathrm{H} \bullet \bullet \mathrm{N} \mathrm{H}$-bond between the bipyridine $\mathrm{Ns}$ and $\mathrm{H}_{t z}$, as commonly observed in similar systems. ${ }^{20,21,22,23}$ Pleasingly, reaction of $o$-Me acetylene $\mathbf{3 b}$ produced rotaxane $\mathbf{4 b}$ in good yield and a significantly enhanced stereoselectivity $(\sim 95 \% d e)$. Indeed, to assign the diastereoselectivity of the reaction, samples of rotaxane $4 \mathrm{~b}$ had to be epimerized so that the ${ }^{1} \mathrm{H}$ NMR resonances of the minor isomer could be identified (see ESI for full details).

Inspection of the ${ }^{1} \mathrm{H}$ NMR spectrum of $\mathbf{4 b}$ (Figure 2aii) led to two observations. Firstly, the resonance attributable to $\mathrm{H}_{t r z}$ appears much closer to that of the same proton in the corresponding axle (Figure 2ai, $\Delta \delta=0.5 \mathrm{ppm}$ ) than observed for rotaxane $4 \mathrm{a}$. Secondly, the diastereotopic methylene ether protons $\mathrm{H}_{\text {ether }}$ appear as a well separated $\left(\Delta \delta=0.7 \mathrm{ppm}\right.$ ) pair of doublets in the ${ }^{1} \mathrm{H}$ NMR spectrum of $\mathbf{4} \mathbf{b}$, whereas in the case of $4 \mathbf{a}$ a much smaller separation was observed $(\Delta \delta=0.2 \mathrm{ppm})$. This suggests that the mechanical stereochemistry of rotaxanes 4 is well expressed around the ether methylene unit (the same signals in the corresponding axles appear as a singlet) and that this effect is larger for $\mathbf{4 b}$ than $\mathbf{4 a}$. In contrast, aliphatic protons associated derived from the azide component are more shielded in rotaxane $\mathbf{4 a}$ than $\mathbf{4 b}$ relative to the corresponding non-interlocked axle. 
Figure 2. (a) Observation of the effect of an o-Me substituent on the diastereoselectivity of mechanical bond formation and the co-conformational bias in rotaxanes $4^{a, b}$ (b) Partial ${ }^{1} \mathrm{H}$ NMR of (i) the noninterlocked axle of rotaxane $\mathbf{4 b}$, (ii) rotaxane $\mathbf{4 b}$, (iii) rotaxane $\mathbf{4 a}$, (iv) the non-interlocked axle of rotaxane 4a (colors and atom labels as in (a) with the exception of macrocycle protons which are shown in blue; correlations shown for protons $\mathrm{H}_{t z}, \mathrm{H}_{\text {ether }}$ and aliphatic protons derived from the azide unit). (c) Solid state structure of $\mathbf{4 b}$ in sticks and spacefilling representations with selected intercomponent interactions indicated (substituted benzyl ether unit omitted for clarity, colors as in (a)).

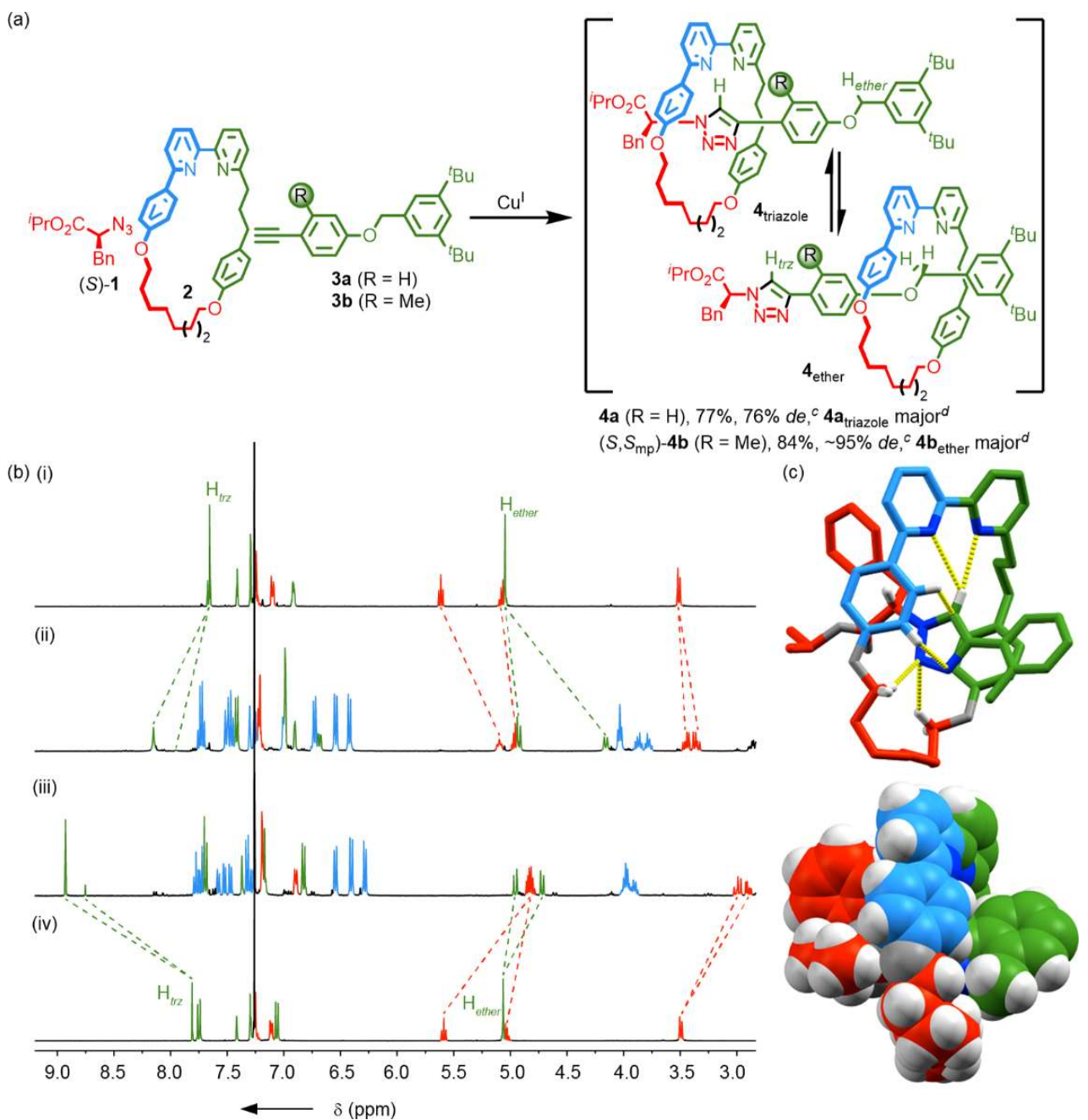

${ }^{a}$ The absolute stereochemistry shown for rotaxanes $\mathbf{4}$ corresponds to the major isomer of $\mathbf{4 b}$ determined by $x$-ray diffraction analysis $\left(S, S_{\mathrm{mp}}\right)$, the major isomer of rotaxane $4 \mathrm{a}$ was not determined. ${ }^{b} \mathrm{Reagents}$ and conditions: [ $\left.\mathrm{Cu}(\mathrm{MeCN})_{4}\right] \mathrm{PF}_{6},{ }^{i} \mathrm{Pr}_{2} \mathrm{EtN}, \mathrm{CH}_{2} \mathrm{Cl}_{2}$, rt, $16 \mathrm{~h}$. ${ }^{6}$ Determined by ${ }^{1} \mathrm{H}$ NMR analysis of the crude reaction products. ${ }^{d}$ Determined by ${ }^{1} \mathrm{H}$ NMR analysis of the purified products.

Taken together, these observations are consistent with rotaxanes 4 existing as a mixture of rapidly exchanging co-conformations in which the bipyridine macrocycle is localized around either the triazole $\left(\mathbf{4}_{\text {triazole }}\right)$ or ether $\left(\mathbf{4}_{\text {ether }}\right)$ moiety of the axle (Figure $2 \mathrm{a}$ ), presumably in both cases through $\mathrm{C}-\mathrm{H} \cdots \cdots \mathrm{N} \mathrm{H}$ bonds, augmented by other weak interactions $(\pi-\pi, \mathrm{CH}-\pi)$. Indeed, by comparing the difference in triazole chemical shifts of the corresponding non-interlocked axles and rotaxanes $4 \mathbf{a}(\Delta \delta=1.2 \mathrm{ppm})$ and $\mathbf{4 b}(\Delta \delta$ $=0.5 \mathrm{ppm}$ ) with the maximum expected chemical shift difference $(\Delta \delta=\sim 2.3 \mathrm{ppm})$ were the triazole station fully occupied (estimated conservatively by considering that the ${ }^{1} \mathrm{H}$ NMR signal attributable to the triazole proton of similar molecules in which the macrocycle is trapped around the triazole unit typically appear 
at $>10 \mathrm{ppm}),{ }^{17}$ we estimate that $\mathbf{4} \mathbf{a}_{\text {triazole }}$ is slightly preferred $(\sim 55 \%)$ whereas $\mathbf{4} \mathbf{b}_{\text {ether }}$ is favored significantly $(\sim 80 \%)$.

The observed difference in co-conformational preference is presumably due to steric clash between the o-Me substituent and the ring which disfavors $\mathrm{H}$-bonding to $\mathrm{H}_{t r z}$. The solid-state structure of $\mathbf{4 b}$ obtained through single crystal $\mathrm{x}$-ray diffraction analysis (Figure $2 \mathrm{c}$ ) is consistent with this proposal as, although the $\mathbf{4} \mathbf{b}_{\text {triazole }}$ co-conformation was found in the solid state, the observed $\mathrm{C}-\mathrm{H} \cdot \bullet \cdot \mathrm{N}$ contacts are much longer than those in other otherwise similar systems $(\mathrm{C}-\mathrm{H} \cdots \cdot \mathrm{N}=2.6$ and 3.0 vs 2.5 and $2.7 \AA) .{ }^{17}$ The space filling representation of the solid-state structure also emphasizes the sterically crowded nature of the triazole station as the macrocycle is seen to be buttressed on one side by the sterically crowded covalent stereogenic center and on the other by the o-Me substituent of the aryl unit. The solid-state structure of 4b also allowed us to assign the absolute stereochemistry of the major isomer to be $\left(S, S_{\mathrm{mp}}\right)-\mathbf{4 b}$.

An "interlocking auxiliary", as introduced by Leigh and Leigh and co-workers ${ }^{24}$ and recently extended by Coutrot, ${ }^{25}$ is a unit that facilitates mechanical bond formation but from which the macrocycle can then be shuttled away from readily to a "receiver" region of the axle, before the interlocking auxiliary unit is removed. The combination of azide 1 with an o-Me aromatic alkyne appears to meet the requirements of both a chiral auxiliary (high stereoselectivity) and interlocking auxiliary (weak non-covalent interactions with the functional group produced) and so could combine these functions as a "chiral interlocking auxiliary".

\section{Demonstration of the chiral interlocking auxiliary concept}

To validate our hypothesis, we investigated the AT-CuAAC reaction of o-Me alkyne 5a with azide (S)-1 and macrocycle 2 with the intention of shuttling the macrocycle onto the unhindered triazole unit prior to removing the chiral interlocking auxiliary to generate rotaxane 8. AT-CuAAC coupling of 5a with (S)-1 and 2 gave rotaxane 6 in excellent isolated yield (80\%). ${ }^{1} \mathrm{H}$ NMR analysis of 6 (Figure S49) suggests that, as expected, the macrocycle is significantly displaced from the hindered triazole; the triazole proton appears at relatively low chemical shift $(\delta=7.60 \mathrm{ppm})$ and the diastereotopic benzylic methylene protons appear as a well separated pair of doublets $(\Delta \delta=0.58 \mathrm{ppm})$. We were unable to ascertain the diastereoselectivity of the reaction at this stage as only one set of signals could clearly be observed.

The PMB protecting group was removed to give rotaxane 7 in which the ring is free to shuttle to the unhindered triazole. Reaction with $\mathrm{TMSCHN}_{2}$ "stoppered" the new axle, trapping the macrocycle over the receiver unit. Finally, addition of $\mathrm{K}_{2} \mathrm{CO}_{3}$ and $\mathrm{MeOH}$ removed the auxiliary fragment to give a crude reaction product containing rotaxane 8 and macrocycle 2 in a $96: 4$ ratio, as judged by ${ }^{1} \mathrm{H}$ NMR analysis of the crude reaction mixture (Figure S56), suggesting that as expected the macrocycle is significantly displaced towards the unhindered triazole unit in of rotaxane 7 under the reaction conditions. Overall, rotaxane 8 was isolated in $65 \%$ yield over 3 chemical steps from 6 (52\% from 5a). Alternatively, starting from carboxylic acid half-axle $\mathbf{5 b}$, rotaxane $\mathbf{7}$ could be produced directly from the AT-CuAAC coupling and converted to rotaxane 8 in $61 \%$ yield over 3 chemical steps from $\mathbf{5 b}$ without isolation of the intermediate structures. CSP-HPLC analysis of the samples of rotaxane 8 produced using our chiral interlocking auxiliary revealed an enantiopurity of $94 \%$ ee, suggesting that the initial AT-CuAAC coupling proceeds in $\sim 94 \%$ de. ${ }^{26}$ Given that the para substituent of the acetylene moiety appears not to strongly influence the diastereoselectivity of the process (both $\mathbf{4 b}$ and $\mathbf{8}$ are produced with similar stereopurity), it is reasonable to assume the orientation of the macrocycle on the axle is the same in both cases. Thus, we tentatively assign the dominant configuration of 8 to be $\left(S_{\mathrm{mp}}\right)-\mathbf{8}$ and by extension, the intermediates to be $\left(S, S_{\mathrm{mp}}\right)-\mathbf{6}$ and $\left(S, S_{\mathrm{mp}}\right)-7 .{ }^{27}$ 
Figure 3. (a) A chiral interlocking auxiliary synthesis of MPC rotaxane $\left(S_{\mathrm{mp}}\right)-8$ using an esterification stoppering strategy. ${ }^{a}$ (b) A chiral interlocking auxiliary synthesis of MPC rotaxane $\left(S_{\mathrm{mp}}\right)$-10 using a crosscoupling stoppering strategy. ${ }^{b}$ (c) A chiral interlocking auxiliary synthesis of phosphine oxide-containing MPC rotaxane $\left(S_{\mathrm{mp}}\right)-12 .^{\circ}$

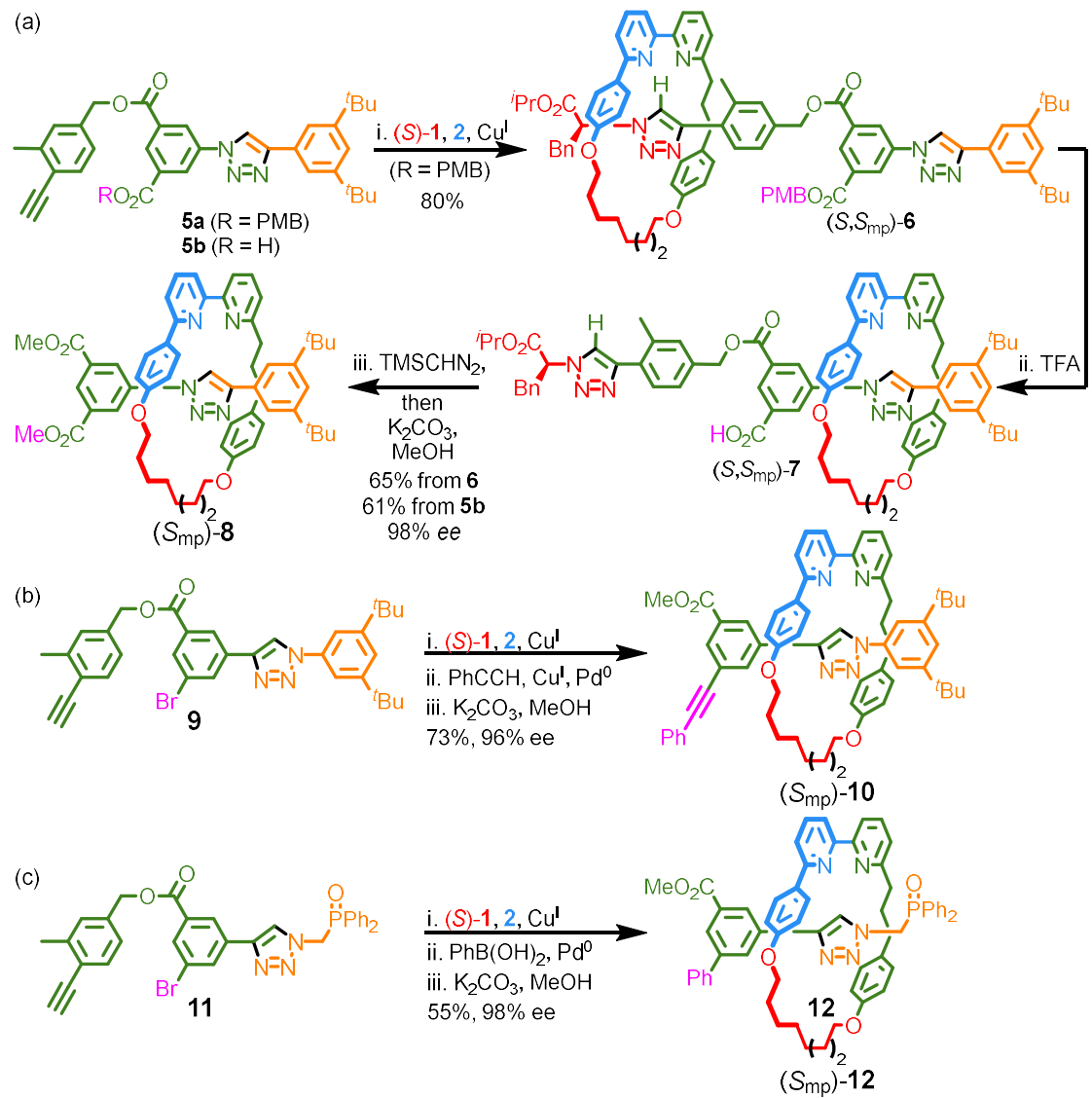

aReagents and conditions: i. [Cu(MeCN) $\left.)_{4}\right] \mathrm{PF}_{6},{ }^{i} \mathrm{Pr}_{2} \mathrm{EtN}, \mathrm{CH}_{2} \mathrm{Cl}_{2}$, rt, $16 \mathrm{~h}(80 \%)$; ii. TFA, $\mathrm{CH}_{2} \mathrm{Cl}_{2}$, rt, 16 h; iii. TMSCHN 2 (2.0 M in hexanes), MeCN, rt, $16 \mathrm{~h}$ then $\mathrm{MeOH}, \mathrm{K}_{2} \mathrm{CO}_{3}, \mathrm{rt}, 3 \mathrm{~h}$ (65\% over two steps, $94 \%$ ee). ${ }^{b}$ Reagents and conditions: i. [Cu(MeCN) $\left.)_{4}\right] \mathrm{PF}_{6},{ }^{i} \mathrm{Pr}_{2} \mathrm{EtN}, \mathrm{CH}_{2} \mathrm{Cl}_{2}$, rt, $16 \mathrm{~h}(89 \%)$; ii. $\mathrm{PhCCH}$, $\mathrm{PdCl}_{2}\left(\mathrm{PPh}_{3}\right)_{2}$, Cul, ${ }^{\mathrm{P} r} \mathrm{NH}_{2}, 110{ }^{\circ} \mathrm{C}, 16 \mathrm{~h}(94 \%)$; iii. $\mathrm{K}_{2} \mathrm{CO}_{3}, \mathrm{MeOH}, \mathrm{CH}_{2} \mathrm{Cl}_{2}$, rt $\left(87 \%, 96 \%\right.$ ee). ${ }^{\circ}$ Reagents and conditions: i. $\left[\mathrm{Cu}(\mathrm{MeCN})_{4}\right] \mathrm{PF}_{6},{ }^{\mathrm{P}} \mathrm{Pr}_{2} \mathrm{EtN}, \mathrm{CH}_{2} \mathrm{Cl}_{2}$, rt, $16 \mathrm{~h}(85 \%)$; ii. $\mathrm{PhB}(\mathrm{OH})_{2}, \mathrm{Pd}(\mathrm{OAc})_{2}, \mathrm{PPh}_{3}$, $\mathrm{K}_{3} \mathrm{PO}_{4} \cdot \mathrm{H}_{2} \mathrm{O}, \mathrm{THF}, 80{ }^{\circ} \mathrm{C}, 16 \mathrm{~h}\left(93 \%, 79: 21\right.$ mixture of co-conformations); iii. $\mathrm{K}_{2} \mathrm{CO}_{3}, \mathrm{MeOH}, \mathrm{CH}_{2} \mathrm{Cl}_{2}$, rt, $16 \mathrm{~h}(70 \%, 98 \% \mathrm{ee})$.

A key advantage of the chiral interlocking auxiliary concept is that, if the azide and o-Me aryl acetylene units are conserved, other details of the structure and reaction sequence can be varied without significantly altering the outcome of the process. For example, if the protected ester functional group of $5 \mathbf{a}$ is replaced by a bromine atom, a similar sequence can be performed in which the stoppering step is achieved using a $\mathrm{Pd}^{0}$ mediated cross coupling; reaction of acetylene $\mathbf{9}$ with macrocycle $\mathbf{2}$ and azide $(S)$ 1 followed by Sonagashira coupling and transesterification to remove the interlocking auxiliary, gave rise to rotaxane $\left(S_{\mathrm{mp}}\right)-10$, once again in similarly excellent yield over 3 steps $(73 \%)$ and enantiopurity $(92 \%$ ee). Similarly, it is relatively trivial to alter the structure of the receiver portion of the axle. Reaction of acetylene 11 with (S)-1 and macrocycle 2, followed by Suzuki cross coupling and cleavage of the auxiliary unit by transesterification, gave MPC phosphine oxide 12 in $60 \%$ overall yield and excellent enantiopurity $(98 \%$ ee $)$. 
Examination of the samples of rotaxanes 10 and 12 immediately after auxiliary cleavage by ${ }^{1} \mathrm{H}$ NMR revealed that the latter contained a lower ratio of the desired product to non-interlocked macrocycle 2 (96: 4 vs $80: 20$ respectively; Figure S108). We cautiously assign this observation to the differing ratio of co-conformations in the corresponding precursor prior to $\mathrm{Pd}^{0}$ mediated cross-coupling. Indeed, whereas in the case of rotaxanes $\left(S, S_{\mathrm{mp}}\right)-\mathbf{4}$ and $\left(S, S_{\mathrm{mp}}\right)-\mathbf{7}$ it was not possible to directly observe the coconformational ratio by ${ }^{1} \mathrm{H}$ NMR as they were in fast exchange compared with the ${ }^{1} \mathrm{H}$ NMR timescale, the $\mathrm{Br}$ substituent of the AT-CuAAC products of $\mathbf{9}$ and $\mathbf{1 1}$ prior to cross coupling is sufficiently bulky to slow the process of co-conformational exchange. ${ }^{1} \mathrm{H}$ NMR analysis revealed signals consistent with an 80 : 20 ratio of receiver : auxiliary co-conformations in the case of the product derived from 11, whereas in the case of the intermediate derived from 9 only trace amounts of a second co-conformation were observed $(>95:<5)$.

\section{Synthesis of "impossible" MPC rotaxanes using a chiral interlocking auxiliary strategy}

Figure 4. Synthesis of "impossible" MPC rotaxanes. (a) Rotaxanes $\left(S_{m p}\right)-13-15$ synthesized using the esterification stoppering approach. (b) Late-stage diversification of rotaxane $\left(S, S_{\mathrm{mp}}\right)-17$ to give MPC rotaxanes $\left(S_{\mathrm{mp}}\right)$-18-20 through a $\mathrm{Pd}^{0}$-mediated stoppering approach.

(a)

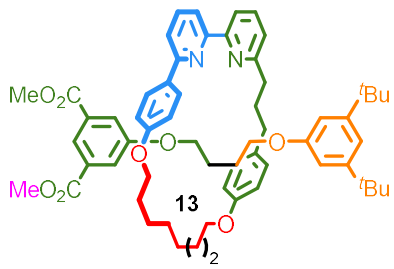

$\left(S_{m p}\right)-13(52 \%$ over 4 steps, $94 \%$ ee $)$

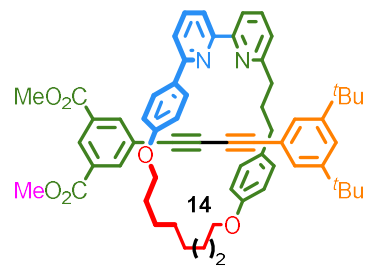

$\left(S_{\mathrm{mp}}\right)-14$ (24\% over 4 steps, $94 \%$ ee)

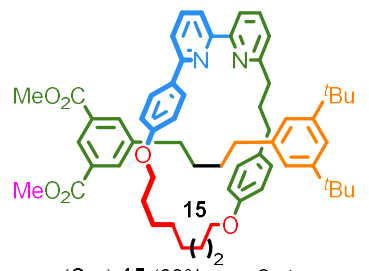

$\left(S_{\text {mp }}\right)$-15 (28\% over 3 steps from phenyl ester, $93 \%$ ee)

(b)
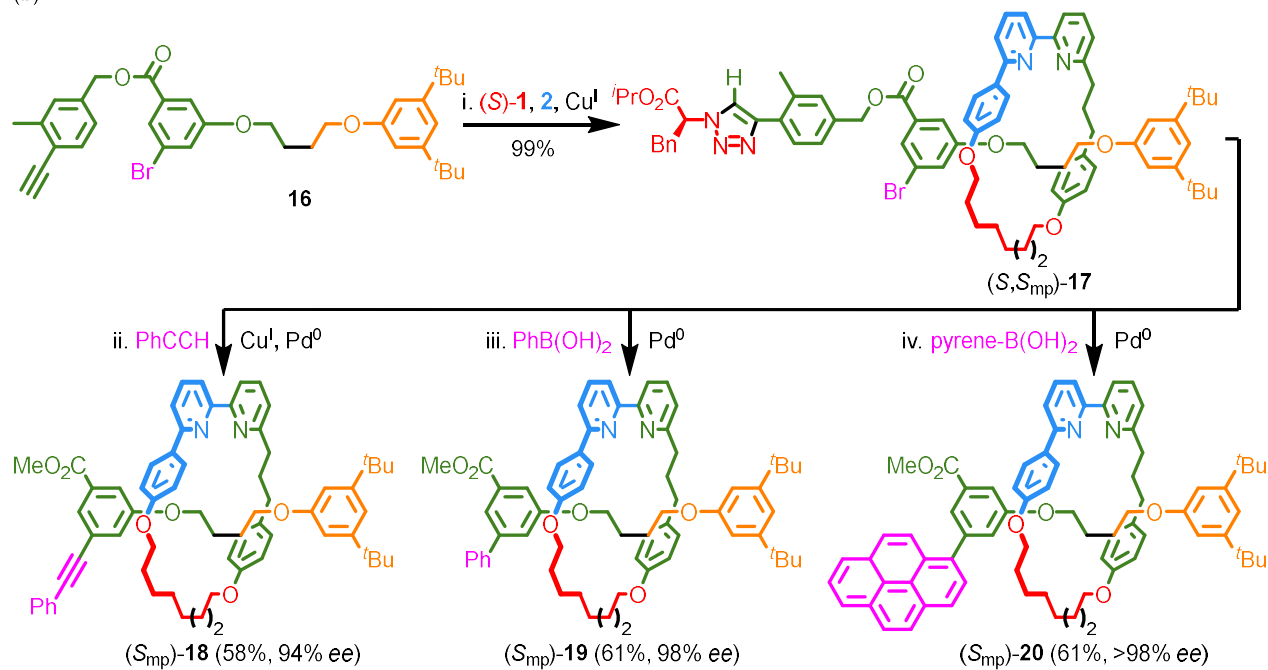

Reagents and conditions: i. [Cu(MeCN $\left.)_{4}\right] \mathrm{PF}_{6},{ }^{i} \mathrm{Pr}_{2} \mathrm{EtN}, \mathrm{CH}_{2} \mathrm{Cl}_{2}$, rt, 16 h (99\%); ii. $\mathrm{PhCCH}, \mathrm{PdCl}_{2}\left(\mathrm{PPh}_{3}\right)_{2}$, Cul, ${ }^{i} \mathrm{Pr}_{2} \mathrm{NH}, 110{ }^{\circ} \mathrm{C}, 16 \mathrm{~h}$, then aqueous work up, then $\mathrm{MeOH}, \mathrm{K}_{2} \mathrm{CO}_{3}, \mathrm{CH}_{2} \mathrm{Cl}_{2}$, rt $(58 \%$, $94 \%$ ee $)$; iii. $\mathrm{PhB}(\mathrm{OH})_{2}, \mathrm{Pd}(\mathrm{OAc})_{2}, \mathrm{PPh}_{3}, \mathrm{~K}_{3} \mathrm{PO}_{4} \cdot \mathrm{H}_{2} \mathrm{O}$, THF, $80{ }^{\circ} \mathrm{C}, 16 \mathrm{~h}$ (aqueous work up), then $\mathrm{MeOH}, \mathrm{K}_{2} \mathrm{CO}_{3}$, $\mathrm{CH}_{2} \mathrm{Cl}_{2}$, rt $\left(61 \%, 98 \%\right.$ ee); iv. $\mathrm{PhB}(\mathrm{OH})_{2}, \mathrm{Pd}(\mathrm{OAc})_{2}, \mathrm{PPh}_{3}, \mathrm{~K}_{3} \mathrm{PO}_{4} \cdot \mathrm{H}_{2} \mathrm{O}$, THF, $80{ }^{\circ} \mathrm{C}, 16 \mathrm{~h}$ (aqueous work up), then $\mathrm{MeOH}, \mathrm{K}_{2} \mathrm{CO}_{3}, \mathrm{CH}_{2} \mathrm{Cl}_{2}$, rt $(61 \%$, >98\% ee)

The results above demonstrate that the combination of azide 1 and an o-Me aryl acetylene benzylic ester fragment can act as a chiral interlocking auxiliary. They also demonstrate, as in the synthesis of rotaxanes $\left(S_{\mathrm{mp}}\right)$-10 and $\left(S_{\mathrm{mp}}\right)$-12, that the structure of the receiver unit can significantly alter the efficiency of the shuttling process integral to this methodology. However, rotaxanes 8, 10 and 2 can all be synthesized directly using the AT-CuAAC reaction, albeit in racemic form, indeed that is how these samples were prepared for CSP-HPLC analysis. Given that one of the benefits of the interlocking 
auxiliary concept is that it can allow access to targets that do not contain motifs that facilitate mechanical bond formation, we set out to explore the limits of our methodology through the synthesis of such socalled "impossible rotaxanes", systems in which there is no significant interaction between axle and macrocycle.

Using a precursor analogous to alkyne $\mathbf{5 a}$ in which the triazole receiving unit is replaced with a simple alkyl bis-ether fragment gave rotaxane $\left(S_{\mathrm{mp}}\right)$-13 (see ESI for full details). In this case the macrocycle is presumed to interact with the receiving unit through $\mathrm{C}-\mathrm{H} \cdots \cdot \mathrm{N}$ hydrogen bonds between the polarized ether protons and the bipyridine unit. Despite the comparatively weak nature of this interaction, ${ }^{1} \mathrm{H}$ NMR analysis of the crude reaction mixture after transesterification revealed a $88: 12$ ratio of rotaxane $\left(S_{\mathrm{mp}}\right)$ 13 to macrocycle 2. Overall, rotaxane $\left(S_{\mathrm{mp}}\right)-13$ was isolated in $52 \%$ yield and $94 \%$ ee over 4 steps.

To probe the effect of reducing the strength of the interactions between the receiving unit and the macrocycle further, we investigated the synthesis of rotaxane $\left(S_{\mathrm{mp}}\right)-14$ in which the di-yne receiving unit presents no significant attractive interactions to the macrocycle at all. Surprisingly, the crude product mixture after transesterification revealed a ratio of $\left(S_{\mathrm{mp}}\right)-14: 2$ of $\sim 2: 3$, suggesting that even in this case the receiving unit competes effectively for the macrocycle, allowing di-yne rotaxane $\left(S_{\mathrm{mp}}\right)-14$ to be isolated in $24 \%$ overall yield and $94 \%$ ee after a challenging final purification.

Similarly, rotaxane $\left(S_{\mathrm{mp}}\right)-15$, in which the macrocycle encircles a simple alkyl chain could be synthesized, albeit in a much lower isolated yield of just $11 \%$ in the final step, due in part to a challenging final purification and in part, based on the ratio of $\left(S_{\mathrm{mp}}\right)-15: 2(2: 3)$ in the crude product of transesterification, the co-conformational bias favoring the auxiliary unit. This could be improved when a phenolic ester link was used in place of the benzylic ester; in this case rotaxane $\left(S_{\mathrm{mp}}\right)-\mathbf{1 5}$ was isolated in $28 \%$ yield after transesterification to remove the auxiliary unit and $\left(S_{\mathrm{mp}}\right)-15$ was the dominant species observed in the crude reaction mixture $\left(\left(S_{\mathrm{mp}}\right)-15: 2=3: 2\right)$. This suggests that the benzylic methylene acts as a competing station for the macrocycle through $\mathrm{C}-\mathrm{H} \cdots \cdots \mathrm{N}$ H-bonding, consistent with our previous observations in the case of $\mathbf{4 b}$.

Finally, we extended our synthesis of impossible MPC rotaxanes by demonstrating the use of our $\mathrm{Pd}^{0}$ mediated cross-coupling stoppering approach in the late-stage diversification of a common building block. ${ }^{28}$ Rotaxane $\left(S, S_{\mathrm{mp}}\right)$-17 was synthesized from the corresponding alkyne block by reaction with macrocycle 2 and azide (S)-1 in excellent yield and subjected to a Sonagashira cross-coupling with phenyl acetylene to give conjugated rotaxane $\left(S_{\mathrm{mp}}\right)-18$, Suzuki cross-coupling with $\mathrm{PhB}(\mathrm{OH})_{2}$ to produce $\left(S_{\mathrm{mp}}\right)$-19, and Suzuki cross-coupling with pyrene-boronic acid to give rotaxane $\left(S_{\mathrm{mp}}\right)$-20 all in good yield and enantiopurity ( $\geq 94 \%$ ee in all cases).

\section{Stereodivergent synthesis using a single set of building blocks}

As demonstrated above, our chiral interlocking auxiliary strategy notionally achieves the threading of a macrocycle onto an axle in a fixed orientation. Perhaps less obviously, the absolute configuration of the final product depends on which side of the receiver unit the interlocking auxiliary is tethered to. Thus, in theory, it is possible to synthesize both enantiomers of an MPC rotaxane using a single stereoisomer of the auxiliary by selecting which side the ring is threaded from.

To demonstrate this principle, we extended our chiral interlocking auxiliary approach to employ a direct aminolysis reaction as this avoids the need for a stoppering step to trap the macrocycle on the receiver region. Using this approach, rotaxane 22a was produced over two steps using a grafting reaction ${ }^{24,29}$ to replace the auxiliary unit. Precursor 21 a was produced in $80 \%$ yield by reaction of the corresponding alkyne with azide 1 and macrocycle 2. The macrocycle in 21a is free to explore the length of the axle and, as expected, ${ }^{1} \mathrm{H}$ NMR analysis (see ESI) suggested the preferred co-conformation is that in which the ring encircles the alkyl chain of the ester portion of the axle, presumably due to $\mathrm{C}-\mathrm{H} \bullet \bullet \cdot \mathrm{N} \mathrm{H}$-bonding interactions. Reaction of $\mathbf{2 1 a}$ with 3,5-di- ${ }^{\mathrm{B}} \mathrm{Bu}$ benzylamine (25a) gave rotaxane $\mathbf{2 2 a}$ in excellent selectivity for the interlocked product; a ratio of $84: 16(22 a: 2)$ was observed by ${ }^{1} \mathrm{H}$ NMR analysis of the crude 
aminolysis reaction product. Interestingly, when a shorter alkyl ester receiver unit was used (21b) the aminolysis reaction proceeded more slowly than that of $\mathbf{2 1 a}$ and with very poor selectivity for the interlocked product (21b : $\mathbf{2} \sim 96: 4$ ), despite ${ }^{1} \mathrm{H}$ NMR analysis of both $\mathbf{2 1 a}$ and $\mathbf{2 1 b}$ suggesting that the preferred co-conformation is that in which the ring encircles the receiver portion of the axle $\left(\mathrm{H}_{t r z}\right.$ appears at $\sim 8 \mathrm{ppm}$ in both cases, suggesting $2 \mathbf{1}_{\mathrm{trz}}: \mathbf{2} \mathbf{1}_{\text {ester }} \sim 10: 1$ ). Thus, we attribute the poor selectivity for $\mathbf{2 2 b}$ to an unfavorable kinetic resolution process; as the phenolic ester unit is much more hindered when the macrocycle occupies the receiver unit, the reaction preferentially occurs when the ring is shuttled away. Based on this, although the direct aminolysis grafting approach provides a synthetically simpler route to MPC rotaxanes than the "stoppering" routes presented above, it is more prone to steric effects, as might be expected given that the macrocycle is proximal to the reacting ester functionality in the key step.

Figure 5. (a) Chiral interlocking auxiliary synthesis of MPC rotaxanes $\left(S_{\mathrm{mp}}\right)-22$ using an aminolysis grafting reaction. ${ }^{a}$ (b) Synthesis of both enantiomers of rotaxane MPC 28 by varying the order of amide bond forming steps. ${ }^{b}$
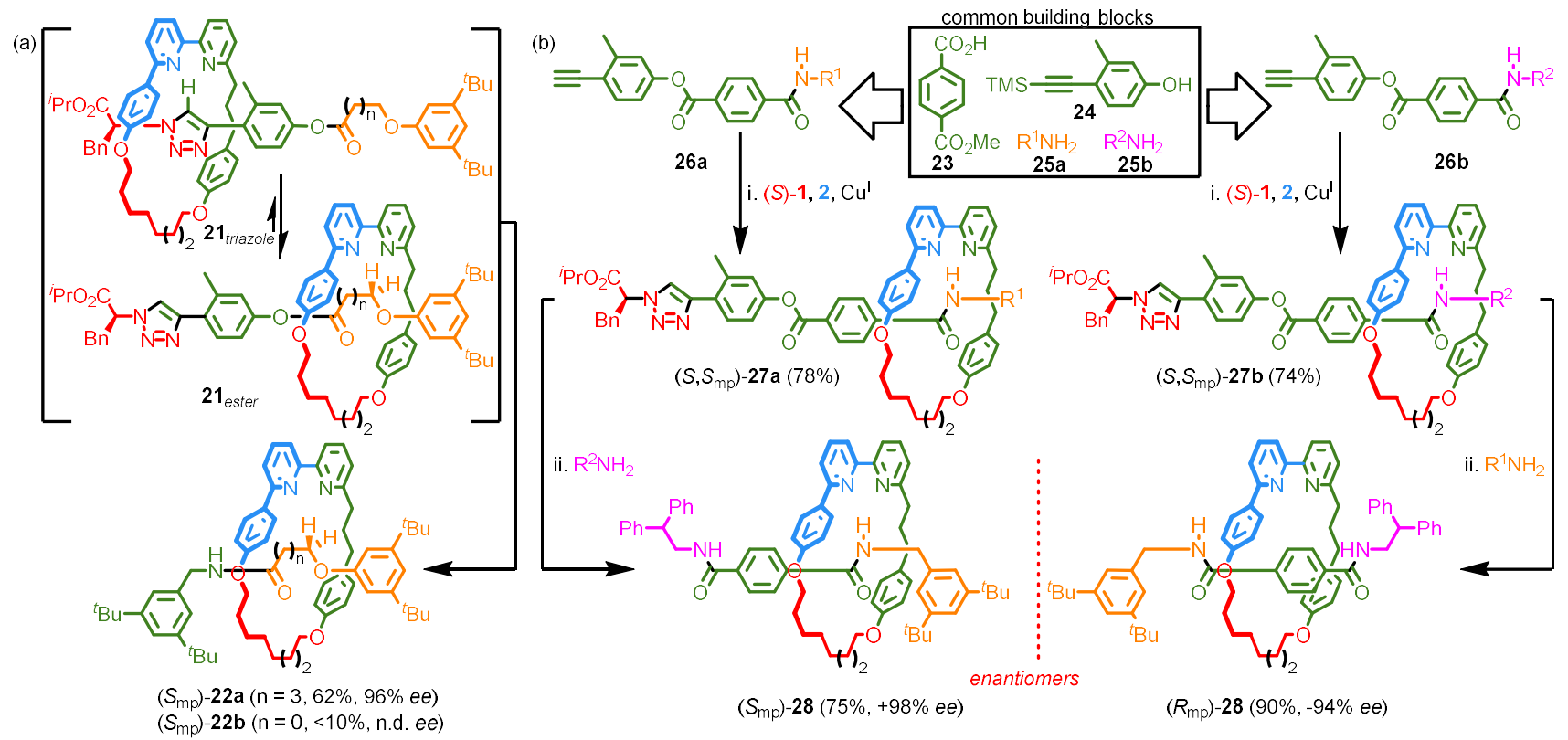

${ }^{a}$ Reagents and conditions: 25a, $\mathrm{CH}_{2} \mathrm{Cl}_{2}, 60{ }^{\circ} \mathrm{C}, 16 \mathrm{~h}\left(62 \%, 96 \%\right.$ ee). ${ }^{b}$ Reagents and conditions: i. 26a or 26b, (S)-1, 2, [Cu(MeCN) 4 ] $\mathrm{PF}_{6},{ }^{i} \mathrm{Pr}_{2} \mathrm{EtN}, \mathrm{CH}_{2} \mathrm{Cl}_{2}$, rt, $16 \mathrm{~h}$ (78\% for $\left(S, S_{\mathrm{mp}}\right)-\mathbf{2 7 a}$; $71 \%$ for $\left.\left(S, S_{\mathrm{mp}}\right)-\mathbf{2 7 b}\right)$; ii. 25b or $25 \mathrm{a}, \mathrm{CH}_{2} \mathrm{Cl}_{2}, 60{ }^{\circ} \mathrm{C}, 16 \mathrm{~h}\left(75 \%, 98 \%\right.$ ee for $\left(S_{\mathrm{mp}}\right)-28 ; 90 \%, 94 \%$ ee for $\left.\left(R_{\mathrm{mp}}\right)-28\right)$.

Having developed this simple aminolysis approach, we turned our attention to synthesizing both enantiomers of rotaxane $\mathbf{2 8}$ by making use of common axle building blocks carboxylic acid 23, alkyne $\mathbf{2 4}$ and amines 25. Coupling of $\mathbf{2 3}$ with amine 25a followed by hydrolysis, ester formation and removal of the TMS unit gave half-axle 26a. AT-CuAAC coupling followed by aminolysis with amine 25b gave rotaxane 28. Conversely, if instead carboxylic acid $\mathbf{2 3}$ is coupled first with amine $\mathbf{2 5 b}$ and converted to AT-CuAAC precursor 26b, subsequent AT-CuAAC coupling and aminolysis by amine 25a gave rise to the opposite enantiomer of rotaxane 28. In keeping with this, circular dichroism (CD) and CSP-HPLC analysis the products of these two sequences reveal mirror image spectra (Figure 6a) and enantiopurities of $\geq|94 \%|$ ee (Figure $6 \mathbf{b}$ ) for each enantiomer. 
Figure 6. (a) Circular dichroism spectra of the enantiomers of rotaxane 28. (b) CSP-HPLC analysis of the enantiomers of rotaxane 28 (bottom $\left(R_{\mathrm{mp}}\right)-\mathbf{2 8},+98 \%$ ee; middle racemate; top $\left(S_{\mathrm{mp}}\right)-\mathbf{2 8},-\mathbf{- 9 4 \%}$ ee).
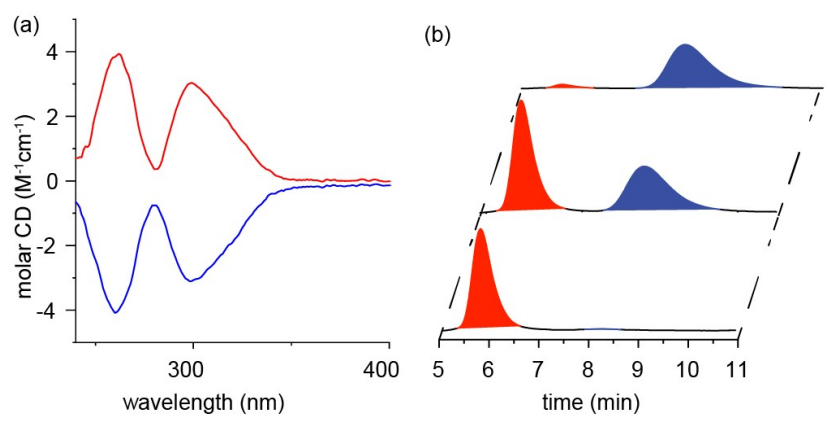

\section{Conclusions}

In conclusion, we have demonstrated a chiral interlocking auxiliary strategy based on the combination of a simple $\alpha$-chiral azide and an o-Me-aromatic acetylene for the synthesis of MPC rotaxanes in which the mechanical bond provides the only stereogenic unit. This approach allows the synthesis of challenging MPC rotaxanes in excellent $(\geq 93 \%$ ee) enantiopurity, including "impossible" rotaxanes where, for example, the macrocycle encircles a simple alkyl chain and, by employing a cross-coupling reaction as the stoppering step, late-stage diversification of a single rotaxane building block can be achieved. Finally, we demonstrate an unusual property of combining molecular motion and mechanical chirality, namely the potential to synthesize both hands of an MPC target using the same starting materials by controlling which end of the axle the macrocycle is effectively threaded on from. Given recent reports of applications of mechanically chiral molecules, and the increased interests in the applications of interlocked molecules in general, we anticipate that these findings will spur further developments in their study and application.

\section{Acknowledgements}

SMG thanks the European Research Council (Consolidator Grant Agreement no. 724987), EPSRC (EP/L016621/1) and Leverhulme Trust (ORPG-2733) for funding and the Royal Society for a Wolfson Research Fellowship. JMS thanks the Royal Society for a Newton International Fellowship. AWH thanks the University of Southampton for a Presidential Scholarship. The authors thank Dr Graham Tizzard of the National Crystallographic Service for helpful discussions around the SC-XRD data.

\section{References}

(1) Bruns, C. J. \& Stoddart, J. F. The Nature of the Mechanical Bond: From Molecules to Machines. (Wiley, 2016).

(2) Erbas-Cakmak, S., Leigh, D. A., McTernan, C. T. \& Nussbaumer, A. L. Artificial Molecular Machines. Chem. Rev. 115, 10081-10206, doi:10.1021/acs.chemrev.5b00146 (2015).

(3) Heard, A. W. \& Goldup, S. M. Simplicity in the Design, Operation, and Applications of Mechanically Interlocked Molecular Machines. ACS Cent. Sci. 6, 117-128, doi:10.1021/acscentsci.9b01185 (2020).

(4) Jamieson, E. M. G., Modicom, F. \& Goldup, S. M. Chirality in rotaxanes and catenanes. Chem. Soc. Rev. 47, 5266-5311, doi:10.1039/c8cs00097b (2018).

(5) Maynard, J. R. J. \& Goldup, S. M. Strategies for the Synthesis of Enantiopure Mechanically Chiral Molecules. Chem 6, 1914-1932, doi:10.1016/j.chempr.2020.07.012 (2020). 
(6) Kaida, Y., Okamoto, Y., Chambron, J. C., Mitchell, D. K. \& Sauvage, J. P. The Separation of OpticallyActive Copper (I) Catenates. Tetrahedron Lett. 34, 1019-1022, doi:Doi 10.1016/S0040-4039(00)774818 (1993).

(7) Yamamoto, C., Okamoto, Y., Schmidt, T., Jager, R. \& Vogtle, F. Enantiomeric resolution of cycloenantiomeric rotaxane, topologically chiral catenane, and pretzel-shaped molecules: Observation of pronounced circular dichroism. J. Am. Chem. Soc. 119, 10547-10548, doi:DOI 10.1021/ja971764q (1997).

(8) Hirose, K., Ukimi, M., Ueda, S., Onoda, C., Kano, R., Tsuda, K., Hinohara, Y. \& Tobe, Y. The Asymmetry is Derived from Mechanical Interlocking of Achiral Axle and Achiral Ring Components Syntheses and Properties of Optically Pure [2]Rotaxanes-. Symmetry 10, 20, doi:10.3390/sym10010020 (2018).

(9) Makita, Y., Kihara, N., Nakakoji, N., Takata, T., Inagaki, S., Yamamoto, C. \& Okamoto, Y. Catalytic Asymmetric Synthesis and Optical Resolution of Planar Chiral Rotaxane. Chem. Lett. 36, 162-163, doi:10.1246/cl.2007.162 (2007).

(10) Tian, C., Fielden, S. D. P., Perez-Saavedra, B., Vitorica-Yrezabal, I. J. \& Leigh, D. A. Single-Step Enantioselective Synthesis of Mechanically Planar Chiral [2]Rotaxanes Using a Chiral Leaving Group Strategy. J. Am. Chem. Soc. 142, 9803-9808, doi:10.1021/jacs.0c03447 (2020).

(11) Denis, M. \& Goldup, S. M. The active template approach to interlocked molecules. Nat Rev Chem 1, 0061, doi:10.1038/s41570-017-0061 (2017).

(12) Tian, C., Fielden, S. D. P., Whitehead, G. F. S., Vitorica-Yrezabal, I. J. \& Leigh, D. A. Weak functional group interactions revealed through metal-free active template rotaxane synthesis. Nat. Commun. 11, 744, doi:10.1038/s41467-020-14576-7 (2020).

(13) Heard, A. W. \& Goldup, S. M. Synthesis of a Mechanically Planar Chiral Rotaxane Ligand for Enantioselective Catalysis. Chem 6, 994-1006, doi:10.1016/j.chempr.2020.02.006 (2020).

(14) Gaedke, M., Witte, F., Anhauser, J., Hupatz, H., Schroder, H. V., Valkonen, A., Rissanen, K., Lutzen, A., Paulus, B. \& Schalley, C. A. Chiroptical inversion of a planar chiral redox-switchable rotaxane. Chem. Sci. 10, 10003-10009, doi:10.1039/c9sc03694f (2019).

(15) Ishiwari, F., Nakazono, K., Koyama, Y. \& Takata, T. Induction of Single-Handed Helicity of Polyacetylenes Using Mechanically Chiral Rotaxanes as Chiral Sources. Angew. Chem. Int. Ed. 56, 14858-14862, doi:10.1002/anie.201707926 (2017).

(16) Eliel, E., Wilen, S. \& Mander, L. Stereochemistry of Organic Compounds. (John Wiley and Sons, Inc., 1994).

(17) Jinks, M. A., de Juan, A., Denis, M., Fletcher, C. J., Galli, M., Jamieson, E. M. G., Modicom, F., Zhang, Z. \& Goldup, S. M. Stereoselective Synthesis of Mechanically Planar Chiral Rotaxanes. Angew. Chem. Int. Ed. 57, 14806-14810, doi:10.1002/anie.201808990 (2018).

(18) Denis, M., Lewis, J. E. M., Modicom, F. \& Goldup, S. M. An Auxiliary Approach for the Stereoselective Synthesis of Topologically Chiral Catenanes. Chem 5, 1512-1520, doi:10.1016/j.chempr.2019.03.008 (2019). 
(19) Aucagne, V., Hanni, K. D., Leigh, D. A., Lusby, P. J. \& Walker, D. B. Catalytic "click" rotaxanes: a substoichiometric metal-template pathway to mechanically interlocked architectures. J. Am. Chem. Soc. 128, 2186-2187, doi:10.1021/ja056903f (2006).

(20) Lahlali, H., Jobe, K., Watkinson, M. \& Goldup, S. M. Macrocycle size matters: "small" functionalized rotaxanes in excellent yield using the CuAAC active template approach. Angew. Chem. Int. Ed. 50, 41514155, doi:10.1002/anie.201100415 (2011).

(21) Lewis, J. E. M., Modicom, F. \& Goldup, S. M. Efficient Multicomponent Active Template Synthesis of Catenanes. J. Am. Chem. Soc. 140, 4787-4791, doi:10.1021/jacs.8b01602 (2018).

(22) Cirulli, M., Kaur, A., Lewis, J. E. M., Zhang, Z., Kitchen, J. A., Goldup, S. M. \& Roessler, M. M. Rotaxane-Based Transition Metal Complexes: Effect of the Mechanical Bond on Structure and Electronic Properties. J. Am. Chem. Soc. 141, 879-889, doi:10.1021/jacs.8b09715 (2019).

(23) Zhang, Z., Tizzard, G. J., Williams, J. A. G. \& Goldup, S. M. Rotaxane Ptll-complexes: mechanical bonding for chemically robust luminophores and stimuli responsive behaviour. Chem. Sci. 11, 18391847, doi:10.1039/c9sc05507j (2020).

(24) Hannam, J. S., Lacy, S. M., Leigh, D. A., Saiz, C. G., Slawin, A. M. \& Stitchell, S. G. Controlled submolecular translational motion in synthesis: a mechanically interlocking auxiliary. Angew. Chem. Int. Ed. 43, 3260-3264, doi:10.1002/anie.200353606 (2004).

(25) Chao, S., Romuald, C., Fournel-Marotte, K., Clavel, C. \& Coutrot, F. A strategy utilizing a recyclable macrocycle transporter for the efficient synthesis of a triazolium-based [2]rotaxane. Angew. Chem. Int. Ed. 53, 6914-6919, doi:10.1002/anie.201403765 (2014).

(26) It should be noted that, because the two diastereomers may have different co-conformational preferences, the link between the de of the initial coupling and the ee of the final product is not direct.

(27) Although we are confident in this assumption, further work to validate the stereoisomer produced using spectroscopic means are ongoing: Koenis, M. A. J., Chibueze, C., Jinks, M., Nicu, V., Visscher, L., Goldup, S. \& Buma, W. J. Vibrational Circular Dichroism Spectroscopy for Probing the Expression of Chirality in Mechanically Planar Chiral Rotaxanes. Chem. Sci., doi:10.1039/d0sc02485f (2020).

(28) Coutrot, F., Waeles, P. \& Gauthier, M. Challenges and Opportunities in the Post-Synthetic Modification of Interlocked Molecules. Angew. Chem. Int. Ed., doi:10.1002/anie.202007496 (2020).

(29) Rowan, S. J. \& Stoddart, J. F. Precision Molecular Grafting: Exchanging Surrogate Stoppers in [2]Rotaxanes. J. Am. Chem. Soc. 122, 164-165, doi:10.1021/ja992919s (2000). 